\title{
RESEARCH
}

\section{Genomic profiling of NETs: a comprehensive analysis of the RADIANT trials}

\author{
James Yao', Abhishek Garg2,†, David Chen ${ }^{3, \dagger}$, Jaume Capdevila4, Paul Engstrom ${ }^{5}$, Rodney Pommier6, \\ Eric Van Cutsem ${ }^{7}$, Simron Singh ${ }^{8}$, Nicola Fazio ${ }^{9}$, Wei $\mathrm{He}^{3}$, Markus Riester ${ }^{2}$, Parul Patel ${ }^{3}$, Maurizio Voi³, \\ Michael Morrissey2, Marianne Pavel ${ }^{10}$ and Matthew Helmut Kulke ${ }^{11}$
}

1 University of Texas MD Anderson Cancer Center, Houston, Texas, USA

${ }^{2}$ Novartis Institutes for Biomedical Research, Cambridge, Massachusetts, USA

${ }_{3}^{3}$ Novartis Pharmaceuticals Corporation, East Hanover, New Jersey, USA

${ }^{4}$ Vall d'Hebron University Hospital, Vall Hebron Institute of Oncology (VHIO), Autonomous University of Barcelona, Barcelona, Spain

${ }^{5}$ Fox Chase Cancer Center, Philadelphia, Pennsylvania, USA

${ }^{6}$ Oregon Health and Science University, Portland, Oregon, USA

7University Hospitals Gasthuisberg/Leuven and KU Leuven, Leuven, Belgium

${ }^{8}$ Odette Cancer Center, Sunnybrook Health Sciences Center, Toronto, Ontario, Canada

${ }^{9}$ European Institute of Oncology, Milan, Italy

10University of Erlangen-Nuremberg, Erlangen, Germany

${ }^{11}$ Dana Farber Cancer Institute, Boston, Massachusetts, USA

Correspondence should be addressed to J Yao: jyao@mdanderson.org

${ }^{\dagger} \mathrm{A}$ Garg and D Chen were employees of Novartis when this research was performed.

\begin{abstract}
Neuroendocrine tumors (NETs) have historically been subcategorized according to histologic features and the site of anatomic origin. Here, we characterize the genomic alterations in patients enrolled in three phase 3 clinical trials of NET of different anatomic origins and assess the potential correlation with clinical outcomes. Whole-exome and targeted panel sequencing was used to characterize 225 NET samples collected in the RADIANT series of clinical trials. Genomic profiling of NET was analyzed along with nongenomic biomarker data on the tumor grade and circulating chromogranin A ( $\mathrm{CgA}$ ) and neuron-specific enolase (NSE) levels from these patients enrolled in clinical trials. Our results highlight recurrent large-scale chromosomal alterations as a common theme among NET. Although the specific pattern of chromosomal alterations differed between tumor subtypes, the evidence for generalized chromosomal instability (CIN) was observed across all primary sites of NET. In pancreatic NET, although the $P$ value was not significant, higher CIN suggests a trend toward longer survival (HR, 0.55, $P=0.077)$, whereas in the gastrointestinal NET, lower CIN was associated with longer survival (HR, $0.44, P=0.0006)$. Our multivariate analyses demonstrated that when combined with other clinical data among patients with progressive advanced NETs, chromosomal level alteration adds important prognostic information. Large-scale CIN is a common feature of NET, and specific patterns of chromosomal gain and loss appeared to have independent prognostic value in NET subtypes. However, whether CIN in general has clinical significance in NET requires validation in larger patient cohort and warrants further mechanistic studies.
\end{abstract}

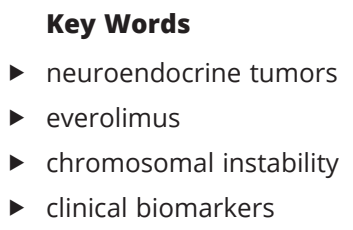

Endocrine-Related Cancer (2019) 26, 391-403 https://erc.bioscientifica.com

https://doi.org/10.1530/ERC-18-0332 (c) 2019 The authors Published by Bioscientifica Ltd. Printed in Great Britain
This work is licensed under a Creative Commons Attribution-NonCommercial-NoDerivatives 4.0 dedternational License ifica.com at 04/26/2023 12:58:25PM 


\section{Introduction}

Neuroendocrine tumors (NETs) are a heterogeneous group of malignancies originating from neuroendocrine cells throughout the body. They are identified most frequently in the gastrointestinal (GI) tract (51\%), lungs (27\%) and pancreas (6\%) (Yao et al. 2008). The prognosis and treatment of patients with NET are currently based on the traditional criteria, including tumor grade, anatomic site of origin and tumor stage (Falconi \& Partelli 2017). While these criteria are broadly associated with the clinical outcomes in large epidemiologic studies, they do not always accurately predict the clinical course of individual patients, nor is it clear that they reflect molecular pathogenesis.

Several studies on relatively small sample cohorts have characterized the genomic profiles of NET. Mutations in the MEN1, DAXX and ATRX genes have been previously reported in approximately 60\% of pancreatic NET (pNET) (Jiao et al. 2011, Scarpa et al. 2017). Scarpa et al. recently identified less frequent mutations in the DNA repair genes MUTYH, CHEK2 and BRCA2 (Scarpa et al. 2017). Further, it was observed that the frequent chromosomal alterations detected by single nucleotide polymorphism (SNP) array including a group with recurrent pattern of whole chromosomal loss, affecting specific chromosomes $(1,2,3,6,8,10$, $11,15,16$ and 22) were similar to our study (Scarpa et al. 2017). However, the authors did not report the loss of chromosome 21 in the cluster. Frequent loss of chromosome 21 has previously been described in detail by Nagano et al. (2007). Mutations in MEN1 and other genes implicated in chromatin remodeling have been reported in bronchial NET (Fernandez-Cuesta et al. 2014). On the other hand, in NET of the small intestine (SI), recurrent mutations in any genes appear to be rare, with mutations in the cyclin-dependent kinase inhibitor (CDKN1B) reported in less than $10 \%$ of cases (Francis et al. 2013).

There are fewer reports exploring the prognostic value of broad genomic profiling on disease outcomes of NET. Loss of heterozygosity (LOH) in chromosome 18 has been previously reported as a potential prognostic biomarker in SI NET (Hashemi et al. 2013, Karpathakis et al. 2016). Mutations in the MEN1, DAXX and ATRX genes in pNET have been shown to be associated with overall survival (OS) (Jiao et al. 2011, Marinoni et al. 2014).

At the same time, histologic markers such as tumor grade, as well as biochemical markers such as circulating levels of chromogranin $\mathrm{A}(\mathrm{CgA})$ and neuron-specific enolase (NSE) have been long known to be associated with disease prognosis in NET, independent of their primary site of origin (van Adrichem et al. 2016, Yao et al. 2016b).

To gain a better understanding of the genomic landscape of NET, we used a combination of nextgeneration sequencing (NGS) strategies to characterize 225 samples collected in the RADIANT series of clinical trials, which were placebo-controlled, phase 3 studies that evaluated the efficacy and safety of everolimus in distinct types of NET. These trials comprised the RADIANT-2 and RADIANT-4 trials in the GI and lung, and the RADIANT-3 trial in the pNET (Pavel et al. 2011, Yao et al. 2011, 2016a). Genomic profiling of a large set of tumor samples from the three distinct primary sites of origin, high-quality nongenomic biomarker data on tumor grade and circulating $\mathrm{CgA}$ and NSE levels from these patients enabled us to comprehensively analyze the clinical utility of performing NGS in NET.

\section{Materials and methods}

\section{RADIANT trials and NGS patient subgroup}

RADIANT-2, RADIANT-3 and RADIANT-4 clinical trials of everolimus in advanced NET have been previously described (Pavel et al. 2011, Yao et al. 2011, 2016a). Tumor samples from a subset of patients in these trials were sequenced and analyzed. In total, 225 tumor samples were successfully sequenced using NGS on whole-exome or targeted cancer panel. Further details on trial designs and number of patients from each trial included in this analysis are listed in Table 1. Each study protocol and associated amendments were reviewed and approved by independent ethics committees or institutional review boards. All patients included in the present analysis consented to evaluation of their archival tumor samples.

\section{DNA extraction and NGS analysis}

Tumor DNA was extracted from a $40 \mu \mathrm{m}$ thick formalinfixed, paraffin-embedded (FFPE) tissue section, which had been histologically confirmed with a tumor purity of $\geq 20 \%$. Among a total of 225 samples, from which, the evaluable NGS data were obtained, 134 libraries were constructed with Illumina TruSeq and sequenced at an average coverage of 100× using Agilent's SureSelect XT Human All Exon V4 kits on an Illumina HiSeq2500 as 100 base-pair paired-end reads. Remaining 91 samples were sequenced at a median coverage of $300 \times$ on a targeted cancer gene 
Table 1 Baseline demographics and disease characteristics.

\begin{tabular}{|c|c|c|c|c|c|c|}
\hline & \multicolumn{2}{|c|}{ RADIANT-2: $\boldsymbol{N}=\mathbf{6 7}\left(16^{b}\right)$} & \multicolumn{2}{|c|}{ RADIANT-4: $\mathbf{N}=\mathbf{9 3}$ (31b) } & \multicolumn{2}{|c|}{ RADIANT-3: $\mathbf{N}=\mathbf{6 5}\left(16^{b}\right)$} \\
\hline & Everolimus $N(\% \mathrm{a})$ & Placebo $N\left(\%^{a}\right)$ & Everolimus $N(\% \mathrm{a})$ & Placebo $N\left(\%^{a}\right)$ & Everolimus $N(\% \mathrm{a})$ & Placebo $N(\% \mathrm{~m})$ \\
\hline NGS/biomarker cohort & $32(48)$ & $35(52)$ & $55(59)$ & $38(41)$ & $29(45)$ & $36(55)$ \\
\hline \multicolumn{7}{|l|}{ Sex } \\
\hline Male & $14(21)$ & $16(24)$ & $19(20)$ & $21(23)$ & $17(26)$ & $22(34)$ \\
\hline Female & $18(27)$ & $19(28)$ & 36 (39) & $17(18)$ & $12(18)$ & $14(22)$ \\
\hline \multicolumn{7}{|l|}{ Age } \\
\hline$<65$ years & $18(27)$ & $25(37)$ & $25(27)$ & $20(22)$ & $20(31)$ & $26(40)$ \\
\hline$\geq 65$ years & $14(21)$ & $10(15)$ & $30(32)$ & 18 (19) & $9(14)$ & $10(15)$ \\
\hline \multicolumn{7}{|l|}{ Race } \\
\hline Caucasian & $29(43)$ & $33(49)$ & $48(52)$ & $26(28)$ & $25(38)$ & $31(48)$ \\
\hline Black & $2(3)$ & $1(1)$ & $3(3)$ & $3(3)$ & $1(2)$ & $2(3)$ \\
\hline Asian & - & - & $3(3)$ & $7(8)$ & $3(5)$ & $3(5)$ \\
\hline Other & $1(1)$ & $1(1)$ & $1(1)$ & $2(2)$ & - & - \\
\hline \multicolumn{7}{|l|}{ Primary/metastatic } \\
\hline Primary & $12(18)$ & $11(16)$ & $33(35)$ & $28(30)$ & $15(23)$ & $16(25)$ \\
\hline Metastatic & $20(30)$ & $24(36)$ & $22(24)$ & $10(11)$ & $14(22)$ & $20(31)$ \\
\hline \multicolumn{7}{|l|}{ Primary site of cancer } \\
\hline Pancreas & - & - & - & - & $29(45)$ & $36(55)$ \\
\hline Lung & $1(1)$ & $4(6)$ & $19(20)$ & $10(11)$ & - & - \\
\hline $\begin{array}{l}\text { SI - small intestine, jejunum, } \\
\text { ileum, duodenum, cecum, CUPc }\end{array}$ & $25(37)$ & $21(31)$ & $26(28)$ & $17(18)$ & - & - \\
\hline Colorectal - rectum, colon & $2(3)$ & $3(4)$ & $7(8)$ & $8(9)$ & - & - \\
\hline $\begin{array}{l}\text { Other GI tissue - stomach, liver, } \\
\text { unannotatedd }\end{array}$ & $4(6)$ & $7(10)$ & $3(3)$ & $3(3)$ & - & - \\
\hline \multicolumn{7}{|l|}{ NGS platform } \\
\hline Targeted panel & $21(31)$ & $16(24)$ & - & - & $21(32)$ & $33(51)$ \\
\hline Whole-exome & $11(16)$ & $19(28)$ & $55(59)$ & $38(41)$ & $8(12)$ & $3(5)$ \\
\hline
\end{tabular}

aSample percentage represents the distribution of demographic and disease subgroups among the biomarker patient cohort with NGS data within each study; bsample percentage represents the percentage of total study population; cCUP denotes that a search for primary was made but not found, and hence, grouped with SI NET; dunannotated grouped with other denotes missing data from case report form.

CUP, cancer of unknown primary; GI, gastrointestinal; NGS, next-generation sequencing; SI NET, small intestinal NET.

panel containing 242 genes at Foundation Medicine Inc. (Cambridge, MA, USA; Supplementary Table 1, see section on supplementary data given at the end of this article). Tumor DNA preparation, library construction, hybrid capture and sequencing were performed as previously described (Frampton et al. 2013). Germline DNA matching 46 pNET samples was additionally sequenced to $50 \times$ on the whole-exome panel. Reads were aligned to hg19 using BWA-MEM 0.7.10 (Li 2013) and marked for duplicates with Picard 1.130. Single nucleotide variants and indels were called with Mutect 1.1.7 (Cibulskis et al. 2013) and Pindel 0.2.5 (Ye et al. 2009), respectively. The normal samples were processed with the same pipeline and merged into a pool of normals after variant calling using GATK CombineVariants (McKenna et al. 2010).

Somatic genetic alterations were reported after filtering the known germline variants present in the public databases and filtering the novel variants present in the 46 normal samples sequenced in this study. Variants were then annotated using SnpEff 4.0 (Cingolani et al. 2012). Nucleotide sequence alterations were queried against known alterations in the catalog of somatic mutations in cancer (COSMIC v70; http://cancer.sanger. ac.uk/cancergenome/projects/cosmic/) and in the SNP database (dbSNP v141; http://www.ncbi.nlm.nih.gov/ $\mathrm{snp} /$ ). Nonsynonymous sequence alterations recorded in COSMIC, but not as common germline in dbSNP, were designated as known somatic mutations; deleterious mutations (nonsense or frameshift) or mutations at splice sites were annotated as likely somatic mutations. Finally, the remaining mutations recorded in dbSNP or in pool of 46 normal samples, and those with no match in either COSMIC or dbSNP, were designated as mutations of unknown significance and were excluded in the subsequent analyses.

Focal copy number variations were assessed, including amplifications (defined as genes with absolute copy number $\geq 6$ copies, adjusted for purity and ploidy) and bi-allelic deletions. Copy number analysis was performed using a targeted cancer panel with copy number tiling probes as per the methods described in previous studies (Frampton et al. 2013, Sun et al. 2014), and on WES 
data using a customized copy number analysis tool, PureCN (Riester et al. 2016). For the latter, coverage was normalized against the pool of normal samples. Samples with the available sequencing data and type of NGS panel are listed in Table 1.

\section{Mutation rate and CIN score}

Mutation rate was calculated only from samples sequenced on the whole-exome panel using PureCN (Riester et al. 2016). Mutation rate was defined as number of mutations predicted to be somatic after adjusting for copy number, purity and non-reference mapping biases, normalized by the length of callable region ( $\geq 30 \times$ coverage and mapping score $\geq 10$ ). Only variants in coding regions with allelic fraction $\geq 0.03$ were counted. The average estimated mutation rate in normal samples was $0.39 / \mathrm{Mb}$ and was subtracted from the tumor averages to correct mutation rates for mosaicism, misclassified variants and remaining sequencing artifacts. Chromosomal instability (CIN) score was defined as the total chromosome length with copy number aberrations normalized by the combined total length of chromosome 1-chromosome 22. CIN scores were reported between 0 and 1 , with CIN score of 0 indicating no chromosomal aberrations, and CIN score of 1 indicating copy number aberrations across the whole genome.

\section{Statistical analysis}

NET subtypes were defined based on K-means clustering of the top three principal components obtained by applying the principal component analysis on nonfocal copy number aberrations reported by our data processing pipeline. Association between clinical features and genomic aberrations was assessed using the Fisher's exact test or the Wilcoxon-Mann-Whitney test. Cox proportional hazard model and log-rank tests were used to assess the association between groups defined by genomic biomarkers and clinical outcome. GI NET from RADIANT-2 and RADIANT-4 trials were pooled together for correlative analysis, and hazard ratios (HRs) were estimated using Cox proportional hazard model stratified by trial. Prognostic analysis was performed by combining patients in all treatment arms and results were reported after adjusting for treatment effect. Multivariate analysis was performed taking into account other known prognostic biomarkers in NET as covariates in the hazard model - including primary site of origin, CgA, NSE and tumor grade.

\section{Results}

Patient demographics and other baseline disease characteristics of the patients are listed in Table 1. We successfully sequenced archival tumor samples from a total of 225 patients, which included 67 patients from RADIANT-2 (30\%), 65 patients from RADIANT-3 (29\%) and 93 patients from RADIANT-4 (41\%) trials. The number of analyzed patients included in the treatment and placebo arms of the three studies is representative of the overall trial population cohort (1:1 for RADIANT-2 and RADIANT-3, and 2:1 for the RADIANT-4 study). Furthermore, no significant difference between treatment and placebo arms was observed in demographics or baseline disease characteristics (e.g. sex, age, race and primary tumor site).

All the 65 pNET samples analyzed in this study were obtained from patients enrolled in the RADIANT-3 trial, providing a large set of pNET with detailed information on patient demographics, treatment regimen, circulating biomarkers and tumor histology for multivariate analysis (Table 1). The number of pNET of primary and metastatic origin was comparable (31 (48\%) and 34 (52\%) unique patient samples, respectively, Table 1). Gastrointestinal NET ( $n=126$ samples) and lung NET ( $n=34$ samples) were pooled together from RADIANT- 2 and RADIANT-4 trials, providing a large cohort of tumor samples with detailed clinical data available for correlative analysis. Gastrointestinal NET were analyzed based on the primary site of cancer - SI sites ( $n=89$ samples from SI, ileum, jejunum, cancer of unknown primary, cecum or duodenum), colorectal sites ( $n=20$ samples from either rectum or colon) and other GI NET ( $n=17$ samples from a mix of various GI tissues, including stomach, liver and unknown primary site of origin; Table 1).

\section{Overall genomic characteristics of NET}

The overall mutation rate, defined as the number of somatic mutations per $\mathrm{Mb}$ of coding region, among the broad cohort of NET was low compared with rates observed in other malignancies and varied little across primary sites. Pancreatic NET and colorectal NET displayed the highest average mutation rates $(1.16 / \mathrm{Mb}$ and $1.08 / \mathrm{Mb}$ ), respectively. SI and lung NET had average mutation rates of $0.82 / \mathrm{Mb}$ and $0.94 / \mathrm{Mb}$, respectively. All NET, however, displayed broad copy number aberrations involving multiple chromosomes. The extent of these broad copy number aberrations among NET was analyzed using a single CIN score, defined as the fraction of total chromosome length with copy number aberrations.

This work is licensed under a Creative Commons Attribution-NonCommercial-NoDerivatives 4.0

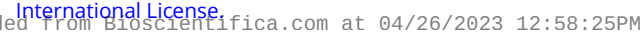


Among the different primary sites, pNET had the highest average CIN (median CIN score $=0.47$ ), while colorectal NET were the least chromosomally unstable tumors (median CIN score $=0.16$ ). SI and lung NET also displayed low median CIN scores in a range similar to colorectal NET (0.16 and 0.19 , respectively).

\section{Genomic landscape of pNET}

MEN1 (43\%), DAXX (28\%), ATRX (11\%), TSC2 (6\%) and PTEN (5\%) were the most frequently mutated genes in pNET. Mutations in the DAXX and ATRX were mutually exclusive, whereas $60 \%$ of $D A X X$ and no ATRX mutation were found to coexist with mutations in the MEN1 gene. The majority of mutations in the MEN1, DAXX and ATRX genes were deleterious $(82 \%$ of all mutations in these three genes were either frameshift indels or small nucleotide changes leading to immature stop codon). Known MTOR/PI3K pathway genes were mutated in $11 \%$ of pNET (four patients with TSC2 mutations and three with PTEN mutations; Fig. 1B). No significant difference in mutation and copy number aberration pattern was observed between primary and metastatic pNET.

Only 11 pNET samples (17\%) had a reported focal copy number loss or amplification $>5$ copies, and CDKN2A and $C D K N 2 B$ were the only two genes with recurring focal copy number aberrations (recurring in $>5 \%$ of samples). However, broad/nonfocal copy number aberrations were observed in $>95 \%$ of pNET, comprising LOH or copy number gain at whole chromosome or at arm level in one or more chromosomes. Unsupervised clustering of nonfocal copy number aberrations suggested the presence of three distinct molecular subtypes of pNET characterized by chromosomal aberration patterns in two mutually exclusive sets of chromosomes; 27 samples in group 1 displayed LOH in chromosome 1, 2, 3, 6, 8, 10, 11, 15, 16, 21 and 22; eight samples in group 2 were characterized by $\mathrm{LOH}$ (with two or more copies of the single chromosome allele) in the same set of chromosomes as group 1, along with the co-occurring copy number gains in a mutually exclusive set of chromosomes comprising chromosome 4, $5,7,9,12,13,14,17,18,19$ and 20. Finally, 30 samples in group 3 were characterized by relatively few copy number abnormalities and absence of any recurring pattern of chromosomal gains or loss (Fig. 1A).

The median CIN scores of samples in groups 1,2 and 3 were $0.47,0.94$ and 0.15 , respectively, based on which, we categorized groups 1 and 2 as CIN high subgroup and group 3 as CIN low subgroup. Among the CIN high subgroups, the samples in groups 1 and 2 had LOH in an identical set of chromosomes, albeit with multiple copies of the same chromosome allele in group 2 samples. Moreover, the mutually exclusive set of chromosomes with wildtype (WT) copy numbers in group 1 display copy number gains in group 2 samples, indicating that group 2 pattern is likely through whole genome duplications, potentially evolved from the samples in group 1 . We, therefore, refer to group 1 as the CIN high-LOH subgroup and group 2 as the CIN high-DUP subgroup.

A strong association was observed between the presence of mutations in the MEN1, DAXX and ATRX genes and the CIN subgroups. All the samples from the CIN high subgroup had mutations in at least one of these three genes compared with only $23 \%$ of samples from the CIN low group, which harbored such mutations (odds ratio, 97.71; $P<9.5 \times 10^{-11}$ Fisher's exact test; Fig. 1B). No particular difference was evident between CIN high-LOH and CIN high-DUP samples in terms of location and type of mutations present in MEN1 and DAXX.

\section{Association of genomic alterations with clinical outcomes in PNET}

We next evaluated whether the CIN-based subgroups or mutations in the MEN1, DAXX and ATRX genes in pNET were associated with any survival outcomes. We also evaluated if these genomic biomarkers have additional prognostic value over known prognostic biomarkers in pNET such as low baseline circulating CgA and NSE levels (Yao et al. 2016b).

Among the three subgroups of pNET, based on CIN patterns, patients in the CIN high subgroups (i.e. group 1-2 together) suggest a trend toward longer median OS of 62.6 months, as compared to the median OS of 37.9 months in patients of the CIN low subgroup (HR, 0.55 ; $95 \%$ confidence interval $(\mathrm{CI}), 0.28-1.07 ; P=0.077$ ) (Fig. 1C and D). Adjusting for baseline circulating $\mathrm{CgA}$ and NSE levels had a minimal impact on the estimated HRs (HR, 0.53; 95\% CI, 0.27-1.05; $P=0.068$ ). Further exploratory analysis of CIN status among low baseline CgA and NSE patients who had better prognosis in NET (Yao et al. 2016b) showed that patients with CIN high status may have further extended OS in both the lowCgA (HR, 0.43; 95\% CI, 0.18-1.03; $P=0.0578$; Fig. 1C) and low-NSE subgroups (HR, $0.6 ; 95 \% \mathrm{CI}, 0.27-1.33 ; P=0.21$; Fig. 1C). However, due to the limited number of events in different groups, statistical significance was not achieved for any of these biomarkers. A limited number of patients with high-baseline CgA and NSE subgroups precluded a similar subgroup analysis of CIN status. Furthermore, 
A

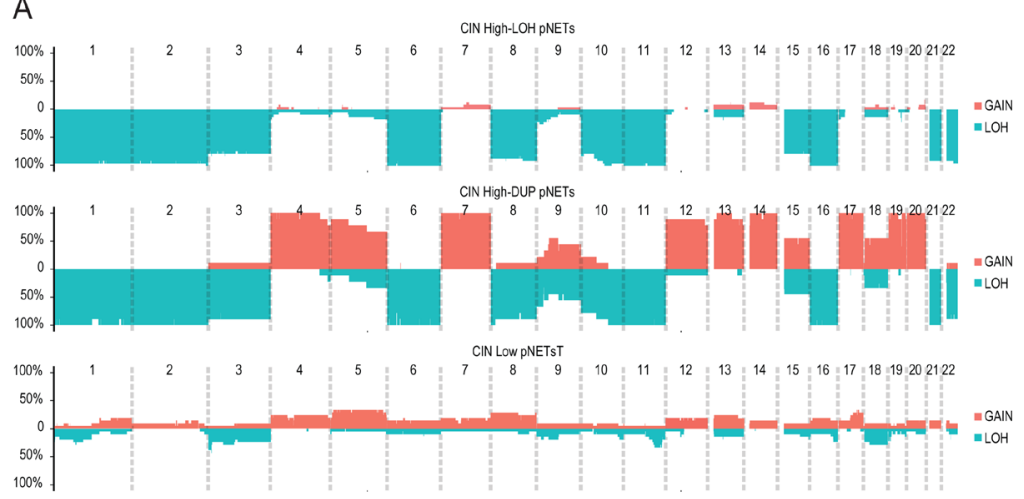

B

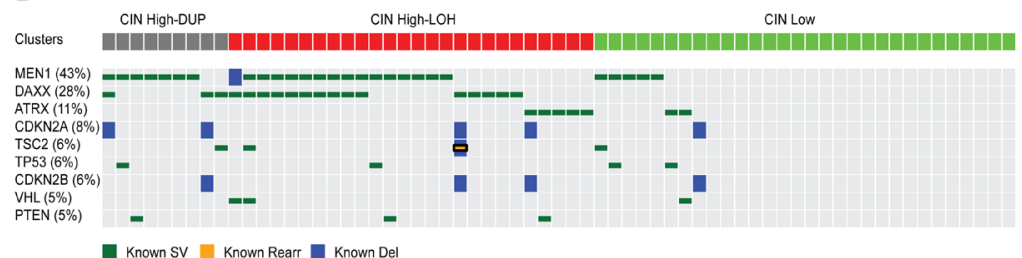

Known SV Known Rearr Known Del

C

Biomarker

CIN status in PNETs

CIN High

GIN LOW

CgA status pNETs Baseline $(\leq 2 \times$ ULN High (> $2 \times$ ULN) CIN status in Iow CgA pNETs CIN High

NSE status PNETs Baseline $(\leq 1 \times$ ULN High (> 1X ULN) CIN status in low NSE pNETs CIN High CIN Low

MEN1/DAXX/ATRX status in PNETs Mutated Wild-type DAXX/ATRX status in PNETs Mutated Wild-type

$\begin{array}{cc}\begin{array}{c}\text { No. of patients } \\ \text { (events) }\end{array} & \begin{array}{c}\text { Median OS } \\ (95 \% \mathrm{Cl})\end{array} \\ 35(18) & 62.6(47.6-\mathrm{NA}) \\ 30(19) & 37.9(17.7-\mathrm{NA}) \\ & \\ 42(21) & 62.6(37.9-\mathrm{NA}) \\ 23(16) & 44.2(13.8-\mathrm{NA}) \\ & \\ 22(9) & \mathrm{NA}(51.1-\mathrm{NA}) \\ 20(12) & 36.6(17-\mathrm{NA}) \\ & \\ 50(26) & 61.2(47-\mathrm{NA}) \\ 14(11) & 15.5(5.8-47.7) \\ 28(14) & 62.6(51.1-\mathrm{NA}) \\ 22(12) & 40.8(17.7-\mathrm{NA}) \\ & \\ 42(23) & 61.2(43.8-\mathrm{NA}) \\ 23(14) & 40.8(23.9-\mathrm{NA}) \\ 25(12) & 62.6(37.9-\mathrm{NA}) \\ 40(25) & 47(35.1-\mathrm{NA})\end{array}$

$40(25)$

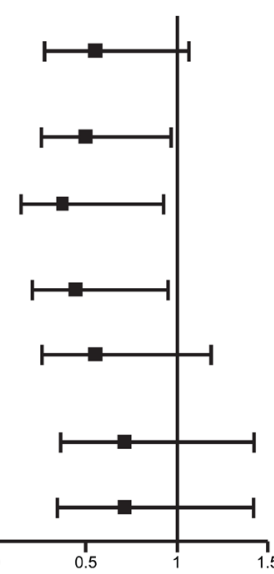

D

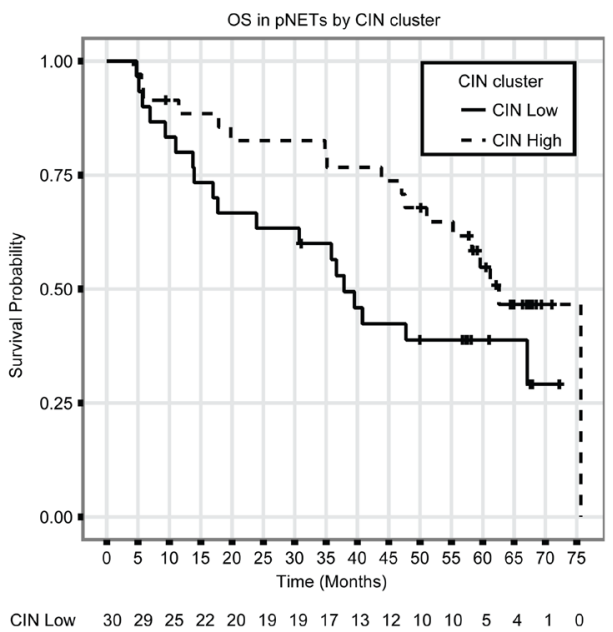

CIN High $\quad \begin{array}{llllllllllllllll}35 & 34 & 31 & 30 & 28 & 28 & 28 & 27 & 26 & 25 & 23 & 21 & 15 & 8 & 2 & 1\end{array}$

Numbers at risk

E

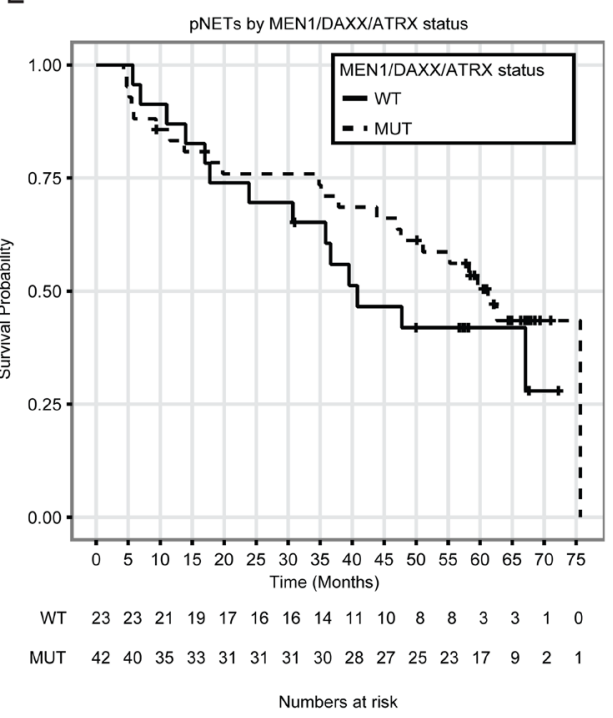

Figure 1

Genomic landscape of 65 pNET and prognostic analysis of biomarkers of OS from RADIANT-3 trial. (A) Three distinct subgroups of pNET identified from unbiased clustering of broad chromosomal copy number gain or loss. The pileup plots show regions of chromosome with copy number aberrations on the $X$-axis and percentage of patients within each subgroup on the $Y$-axis. (B) Mutation profile of patients within each subgroup of patients. Only genes with mutation frequency $>5 \%$ are displayed. (C) Hazard ratio and median OS in subgroup analysis based on genomic biomarkers. (D) Kaplan-Meier plot showing OS among CIN subgroups. (E) Kaplan-Meier plot showing OS among pNET based on mutations status in MEN1, DAXX and ATRX genes. (CIN high group comprising CIN high LOH (group 1) and CIN high DUP (group 2) in panel A). CIN, chromosomal instability; CgA, chromogranin A; LOH, loss of heterozygosity; MUT, sequence mutation or aberrant copy number; NSE, neuron specific enolase; pNET, pancreatic neuroendocrine tumor; OS, overall survival; ULN, upper limit of normal; WT, wild-type.

no association was observed between CIN subgroups and progression-free survival (PFS; HR, 1.32; 95\% CI, $0.73-2.41 ; P=0.36)$.

Mutations in the DAXX and ATRX genes have been previously shown to be associated with longer OS in pNET, both in the presence and absence of mutations in the MEN1 gene (Jiao et al. 2011). In our analysis, patients with mutations in at least one of these three genes did not demonstrate any meaningful improvement in OS compared to patients with WT alleles (HR, 0.71; 95\% CI, $0.36-1.42 ; P=0.34$; Fig. 1C-E). Similarly, no appreciable difference in OS was observed when only the mutation status of the DAXX and ATRX genes is taken into account (HR, 0.71; 95\% CI, 0.35-1.42; $P=0.34$ ). 
Finally, the effect of everolimus on PFS over placebo was similar in patients with both high and low CIN scores; group CIN high: median PFS, 12.5 vs 5.4 months (HR, 0.45; 95\% CI, 0.21-1); group CIN low: median PFS, 10.8 vs 4.6 months (HR, 0.57; 95\% CI, 0.22-1.46), consistent to that observed in the overall study population (median PFS, 11.0 vs 4.6 months; HR, 0.35; 95\% CI, $0.27-0.45, P<0.001)$. Treatment-specific effects in patients with PI3K pathway mutations could not be evaluated, as six of seven patients with mutations in the PI3K pathway genes received placebo. The single everolimus-treated patient with a mutation in the PI3K pathway (a frameshift mutation leading to premature stop codon in TSC2 gene P1771fs*34+) experienced a PFS of 8.1 months.

\section{Genomic landscape and its association with clinical outcomes in lung NET}

MEN1 mutations were present in 6 of 34 lung NET samples (18\%). All six lung NET with MEN1 mutations were loss-offunction mutations accompanied by $\mathrm{LOH}$ in the genomic region coding for MEN1 gene, suggesting potentially a complete loss of MEN1 function in these patients. No other gene was found to be recurrently mutated in lung NET; specifically, known MTOR/PI3K pathway genes such as MTOR, PIK3CA, PTEN, TSC1/2 and PTEN were not found to be mutated in any lung NET samples.

Copy number gain of chromosome 7 was present in $41 \%$ of lung NET, and $\mathrm{LOH}$ in chromosome 3 and chromosome 11 were present in $47 \%$ of lung NET, respectively. Whole chromosome copy number gain/loss was also observed in other chromosomes at a lower frequency, with arm level or whole chromosome gain or $\mathrm{LOH} /$ deletion present in $<25 \%$ of samples. Unsupervised clustering of chromosomal aberration patterns identified three potential clusters of lung NET with median CIN scores of $0.10,0.21,0.71$ in cluster $1(N=19)$, cluster 2 $(N=10)$ and cluster $3(N=5)$, respectively. These clusters were broadly classified into CIN high (comprising samples in clusters 2 and 3 with median CIN score of 0.27 ) and CIN low (comprising samples in cluster 1 with median CIN score of 0.10 ) subgroups.

We obtained only five lung NET samples from the RADIANT-2 trial, and four of these patients received treatment in the placebo arm (Table 1). We, therefore, excluded lung NET from the RADIANT-2 trial in the correlative analysis to avoid study-specific bias. PFS was used as the end point for prognostic assessment of lung NET, as OS has not yet reached the prespecified cutoff in the RADIANT-4 trial population. CIN status was not associated with differences in PFS (HR, 0.95; 95\% CI, 0.36-2.51; $P=0.916$, Fig. 2B and C). However, in our exploratory analysis of the three most frequently occurring broad chromosomal aberrations (copy number gain of chromosome 7, $\mathrm{LOH}$ of chromosome 3 and $\mathrm{LOH}$ of chromosome 11), $\mathrm{LOH}$ in chromosome 3 was found to be significantly associated with PFS. Lung NET patients with WT chromosome 3 (i.e. heterozygous 2 copy of chromosome) displayed more than 2-fold longer median PFS along with $80 \%$ lower risk of disease progression than patients with $\mathrm{LOH}$ or deletion in chromosome 3 (median PFS of 11.2 vs 3.8 months (HR, 0.19; 95\% CI, 0.06-0.56; $P=0.003)$; Fig. $2 \mathrm{~B}$ and $\mathrm{C}$ ). This correlation was still significant after adjusting for tumor grade and baseline circulating levels of CgA and NSE as covariates (HR, 0.24; 95\% CI, 0.08-0.76; $P=0.015$ ). Further subgroup analysis of lung NET was not feasible due to small sample size.

\section{Genomic profiling of GI NET}

Only a handful of genes were found to be recurrently mutated in GI NET. In SI NET, BCOR and CDKN1B were among the most frequently mutated genes (5 of 89 and 4 of 89 samples, respectively). Mutations in both the $B C O R$ and $C D K N 1 B$ genes were mostly loss-of-function mutations, with frameshifts resulting in immature stop codons in 4 of 5 BCOR mutations and 4 of 4 CDKN1B mutations. In colorectal NET, only ATM was found to be mutated recurrently ( 3 of 20 or $15 \%$ of the samples).

No recurring focal copy number amplification/loss was observed in either SI or colorectal NET. However, recurring broad $\mathrm{LOH}$ in chromosome 18 was present in $\sim 50 \%$ of SI and colorectal NET and copy number gains in chromosomes 4, 5, 14 and 20 were observed in $>25 \%$ of SI NET. K-means clustering of broad copy number aberrations identified five distinct clusters in GI NET, each defined by a specific set of chromosomal aberration. Cluster 1 by LOH in chromosome $18(N=75)$; cluster 2 by gain in chromosome 5 and chromosome $7(N=17)$; cluster 3 by gain in chromosome 4 , chromosome 5 , chromosome 14 and chromosome $20(N=18)$; cluster 4 by gain in chromosome 4 , chromosome 5 , chromosome 7, chromosome 14 and chromosome $20(N=13)$; and a smaller cluster 5 comprising three high-ploidy samples was identified, displaying copy number gains across most of the chromosomes (Fig. 3A). As with lung and pNET, these clusters could be broadly dichotomized into CIN high or CIN low subgroups: CIN high group comprising clusters 2 to 5 with median CIN score of 0.26 and CIN low group comprising samples in cluster 1 with median CIN

This work is licensed under a Creative Commons Attribution-NonCommercial-NoDerivatives 4.0 ednternational License ${ }_{1 \text { fica.com at } 04 / 26 / 2023 \text { 12:58:25PM }}$ 
A

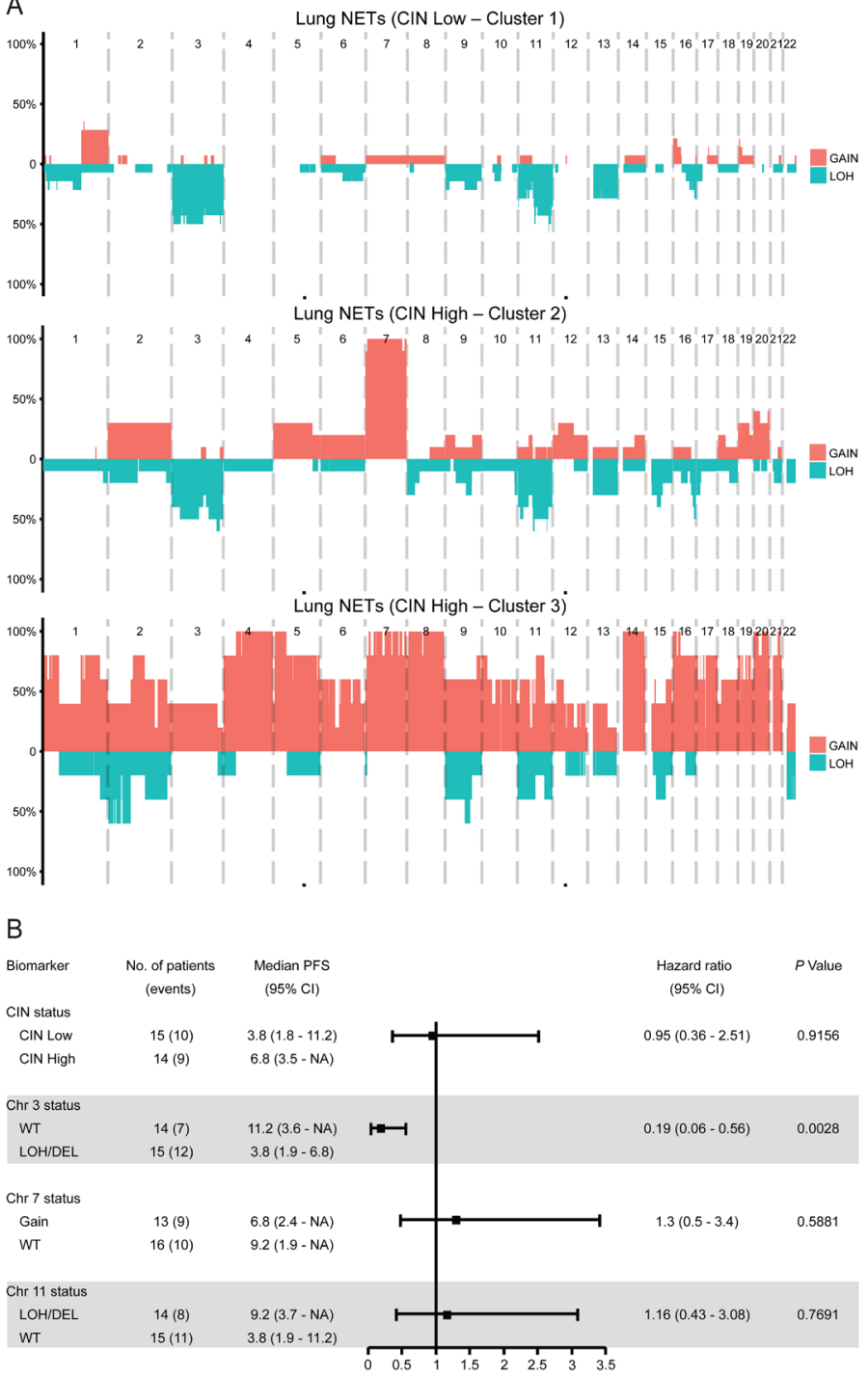

C

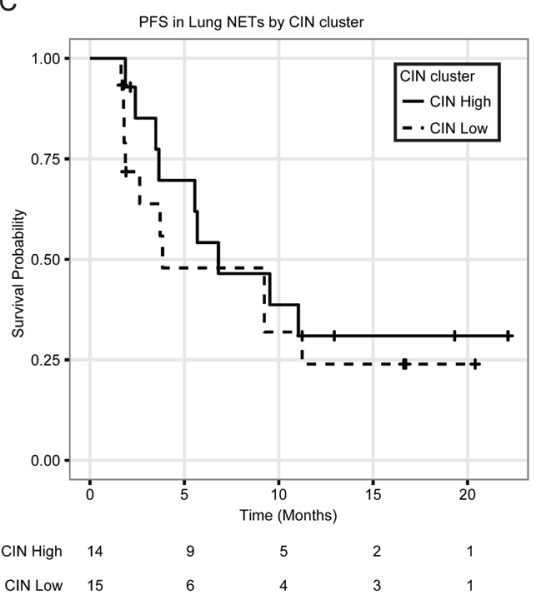

Numbers at risk

D

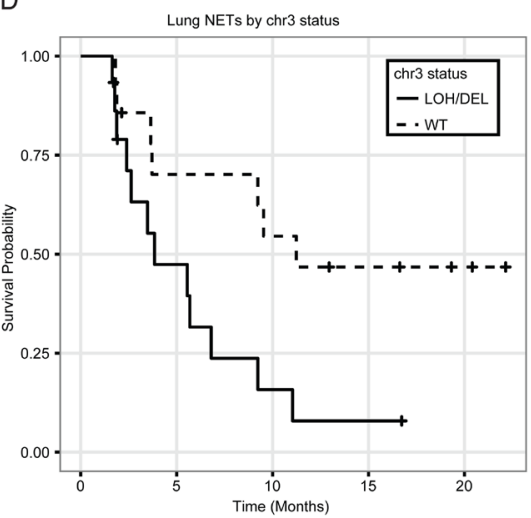

$\begin{array}{llllll}\text { LOH/DEL } & 15 & 6 & 2 & 1 & 0\end{array}$

WT 14

7

\section{Figure 2}

Genomic landscape of 34 lung NET and correlative analysis of prognostic biomarkers of PFS from RADIANT-2 and RADIANT-4 trials. (A) Three distinct subgroups of lung NET identified from unbiased clustering of broad chromosomal copy number gain or loss. (B) Hazard ratio and median PFS in subgroup analysis based on genomic biomarkers from lung NET in RADIANT-4 trial. (C) Kaplan-Meier plot showing PFS among CIN subgroups of lung NET in RADIANT-4 trial. (D) Kaplan-Meier plot showing PFS among lung NET based on copy number status of chromosome 3 in lung NET in RADIANT-4 trial. (CIN high, comprising samples in clusters 2-3 in panel A; CIN low, comprising samples in cluster 1 in panel A). chr, chromosome; CIN, chromosomal instability; LOH/DEL, copy number loss; NET, neuroendocrine tumor; PFS, progression-free survival; WT, wild-type.

score of 0.06. Mutations or copy number aberrations in PI3K pathway genes were present in only 1 of 126 GI NET (an SI NET with focal homozygous deletion of the TSC2 gene in the placebo arm of RADIANT-2).

\section{Association of genomic alterations with clinical outcomes in GI NET}

GI NET samples from RADIANT-2 and RADIANT-4 were analyzed together and HRs were stratified by trial and adjusted for treatment and primary site of origin. PFS was used for prognostic assessment of GI NET, as OS has not yet reached the prespecified cutoff in the RADIANT- 4 trial population.

When NET from all GI tissues were analyzed as a single population, patients in the CIN low group had a significantly longer PFS, with a median PFS that was twice as long and a $56 \%$ lower risk of disease progression vs those in the CIN high group (median PFS of 17.2 months vs 8.2 months (HR, 0.44; 95\% CI, 0.28-0.71; $P=0.0006$ ); Figs 3B and 4). CIN low status is also prognostic of PFS when SI NET are analyzed separately from NET of other GI tissues (Fig. 4). https://erc.bioscientifica.com https://doi.org/10.1530/ERC-18-0332 (c) 2019 The authors Published by Bioscientifica Ltd. Printed in Great Britain
This work is licensed under a Creative Commons Attribution-NonCommercial-NoDerivatives 4.0

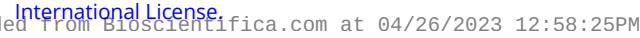


A
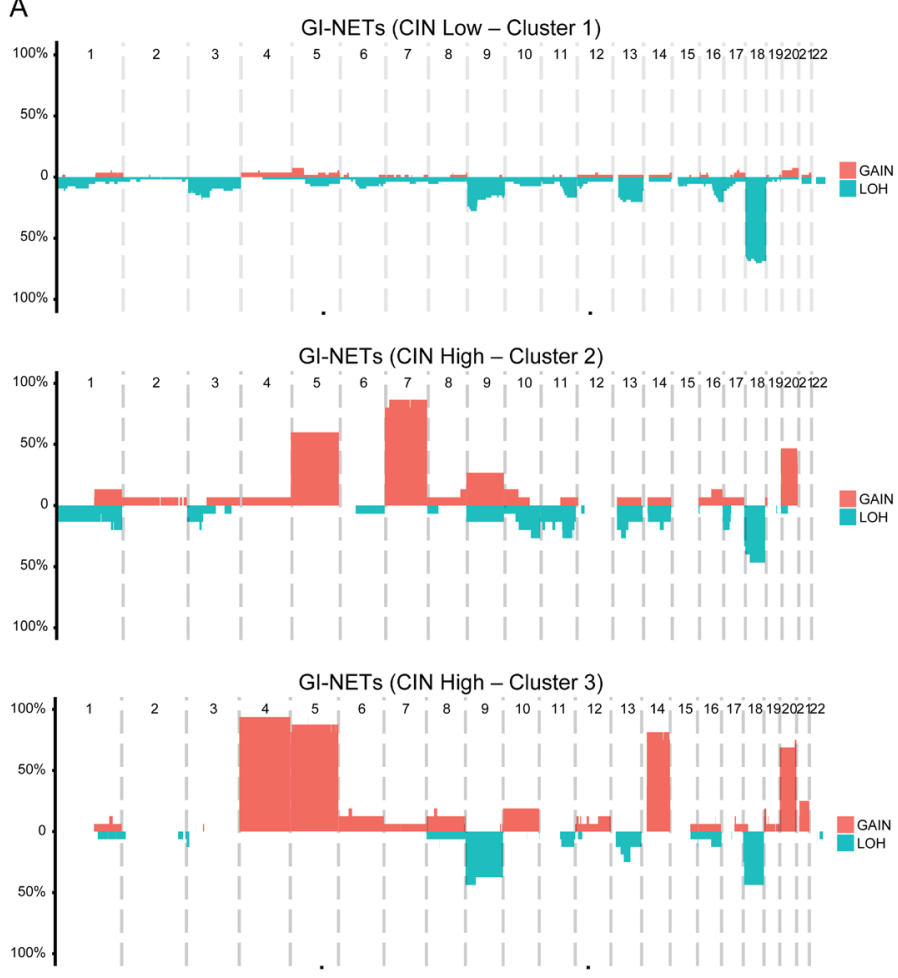

GI-NETs (CIN High - Cluster 4)

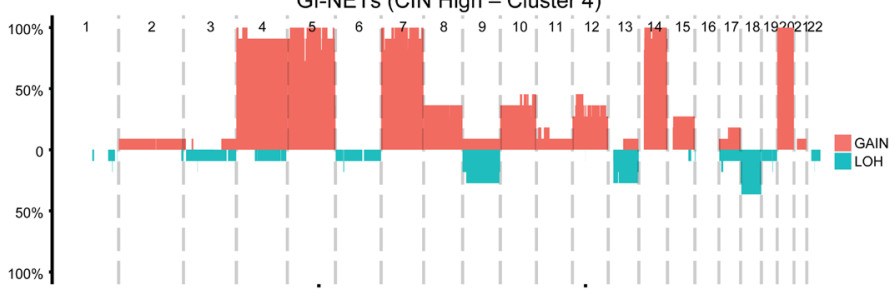

GI-NETs (CIN High - Cluster 5)

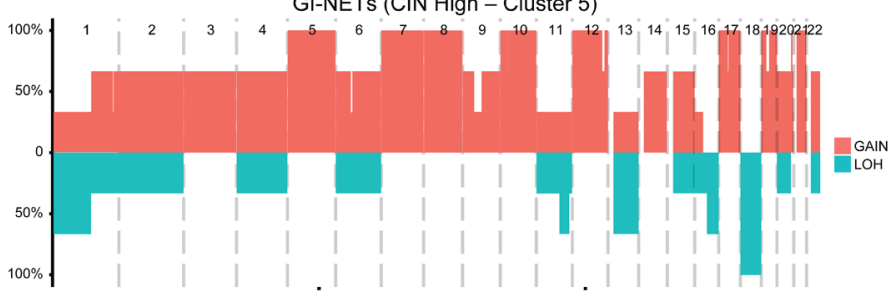

B

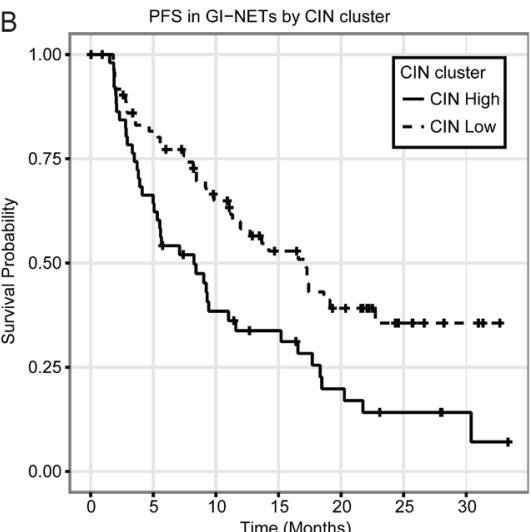

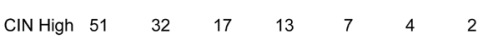

$\begin{array}{lllllll}\text { CIN Low } 75 & 56 & 41 & 28 & 19 & 7 & 3\end{array}$

Numbers at risk

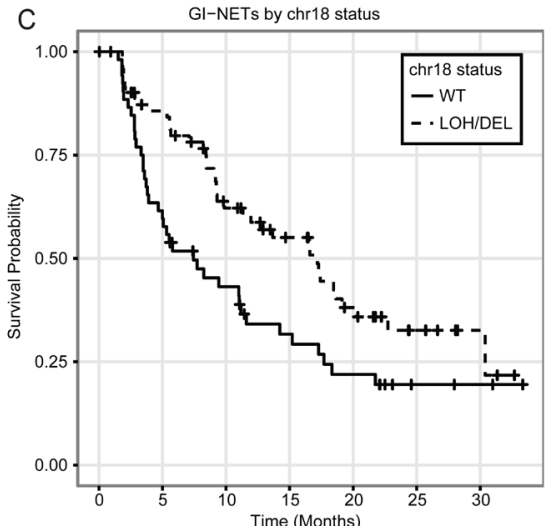

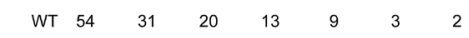
$\begin{array}{llllllll}\text { LOH/DEL } & 72 & 57 & 38 & 28 & 17 & 8 & 3\end{array}$ Numbers at risk

\section{Figure 3}

Genomic landscape of 126 NET from gastrointestinal tissues and prognostic analysis of biomarkers of PFS from RADIANT-2 and RADIANT-4 trials. (A) Five distinct subgroups of GI NET identified from unbiased clustering of broad chromosomal copy number gain or loss. (B) Kaplan-Meier plot showing PFS among CIN subgroups. (C) Kaplan-Meier plot showing PFS among GI NET based on copy number status of chromosome 18. (CIN high group comprising groups 2-5 in panel A; CIN low comprising patients in group 1 in panel A). chr, chromosome; CIN, chromosomal instability; GI, gastrointestinal; LOH/DEL, copy number loss; NET, neuroendocrine tumor; PFS, progression-free survival; WT, wild-type.

Multivariate analysis, taking tumor grade and CgA into account as covariates in the hazard model, showed that CIN-based subgrouping of GI NET is independently prognostic of PFS (HR, 0.48; 95\% CI, 0.30-0.78; $P=0.003$ ). Further exploratory analysis of the subset of GI NET with favorable prognosis based on low tumor grade, low baseline CgA or low NSE shows that the CIN low GI NET patients in these subgroups still displayed significantly longer PFS as compared with the CIN high subgroup (HR, $0.34(P=0.0001)$ in grade 1 ; HR, $0.34(P=0.01)$ in low CgA; and HR, $0.38(P=0.03)$ in low-NSE subgroups of GI NET, respectively; Fig. 4). CIN-based subgroup analysis of GI NET with high-baseline CgA also shows a trend toward better prognosis in CIN low subgroup, although https://erc.bioscientifica.com https://doi.org/10.1530/ERC-18-0332
(C) 2019 The authors Published by Bioscientifica Ltd. Printed in Great Britain
This work is licensed under a Creative Commons Attribution-NonCommercial-NoDerivatives 4.0

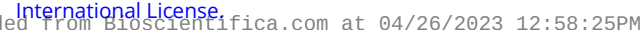




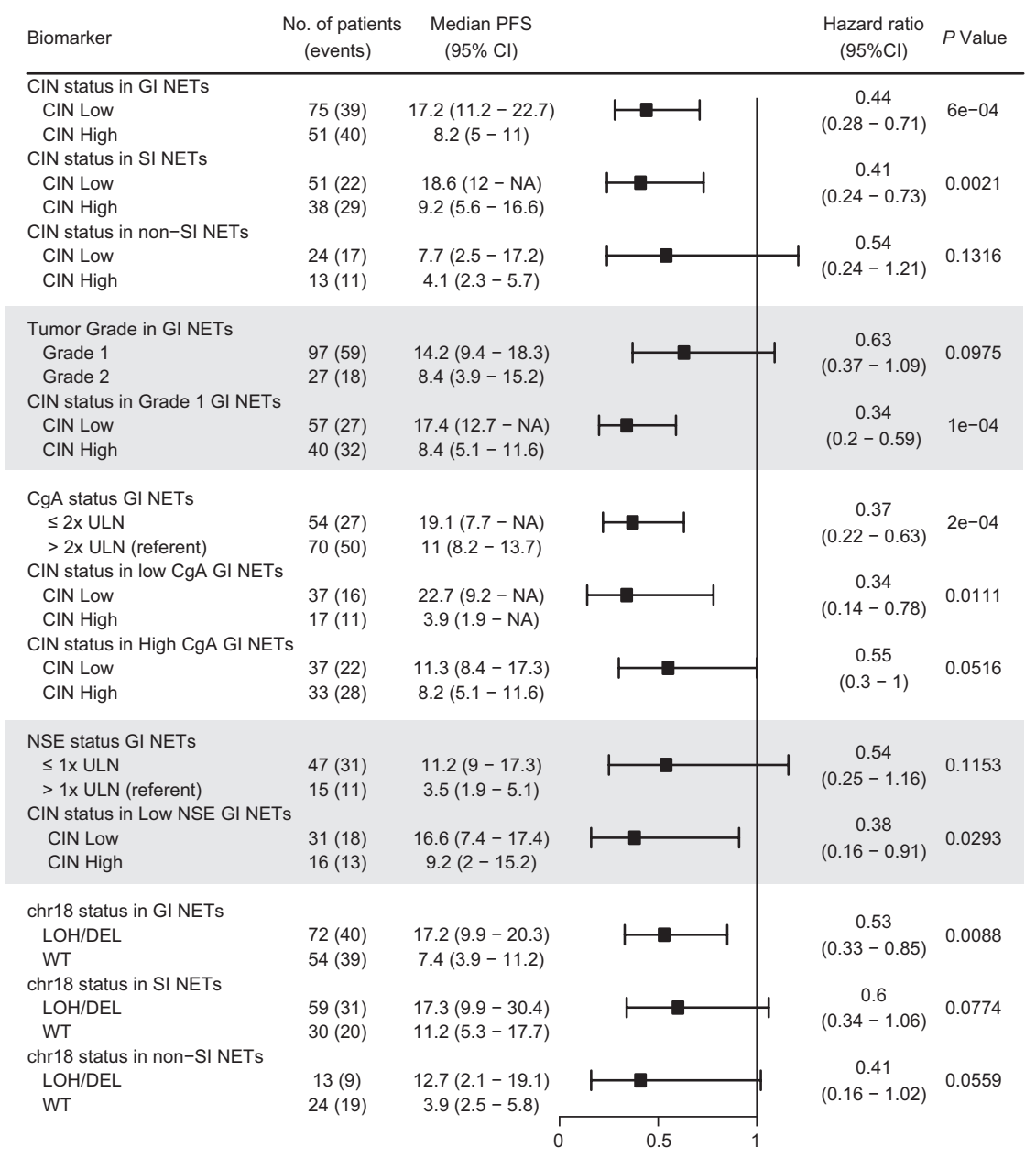

\section{Figure 4}

Prognostic analysis of genomic and nongenomic biomarkers in GI NET from RADIANT-2 and RADIANT-4 trials. Hazard ratio was estimated using Cox proportional hazard model stratified by trial, and median PFS in subgroup analysis based on tumor grade, circulating biomarkers ( $\mathrm{CgA}$ and NSE), and genomic biomarkers. CgA, chromogranin A; chr, chromosome; CIN, chromosomal instability; GI, gastrointestinal; LOH/DEL, copy number loss; NET, neuroendocrine tumor; NSE, neuron specific enolase; PFS, progression-free survival; SI, small intestine; ULN, upper limit of normal; WT, wild-type. not statistically significant (HR, 0.55; 95\% CI, 0.3-1.0; $P=0.05$ ). Further subgroup analysis of CIN status in grade 2 tumors and high NSE levels was not feasible due to the small sample size. In addition, NSE status was only available from the RADIANT-4 study, and therefore, only a subset of GI NET is reported in subgroup analysis based on circulating NSE levels.

$\mathrm{LOH}$ in chromosome 18 has been previously reported as a potential biomarker of good prognosis in SI NET (Hashemi et al. 2013, Karpathakis et al. 2016). Among the GI NET samples profiled in this study, LOH in chromosome 18 was present across all the five clusters identified based on copy number aberrations, and therefore, provides an alternate mechanism for subgrouping GI NET. Gastrointestinal NET with LOH in chromosome 18 displayed significantly longer median PFS than those with WT chromosome 18 (17.2 vs 7.4 months (HR, 0.53 ; 95\% CI, 0.33-0.85; $P=0.009$ ); Figs $3 \mathrm{C}$ and 4). When chromosome 18 status in SI and non-SI NET were analyzed separately, non-SI NET with WT chromosome 18 displayed the smallest median
PFS of 4 months among all the subgroups (95\% CI, 2.5-5.8 months; Fig. 4).

\section{Discussion}

In this study, we performed genomic profiling on 225 tumor samples from patients with NET enrolled in the RADIANT series of phase 3 clinical trials. Detailed genomic profiles together with carefully annotated clinical data and patient demographic information from one of the largest and most diverse cohort of NET presented a unique opportunity to characterize the genomic landscape of NET and correlate these findings with clinical outcomes. Our data suggest that genomic biomarkers based on broad chromosomal aberration patterns in NET may add further value to frequently used histopathology and circulating protein biomarkers for defining a more accurate prognosis of NET in the clinic.

In our analysis, low CIN in GI NET was found to be significantly associated with improved PFS and 
appeared to provide additional prognostic value when combined with other nongenomic biomarkers such as tumor grade and circulating CgA and NSE. While these conclusions are derived only from a small sample size and require validation in a larger cohort, the data presented here highlights the potential clinical utility of genomic profiling in patients with NET from GI tissues. In accordance with previously reported results, our data highlighted the remarkably low frequency of recurring mutations in GI NET. Recurring mutations in BCOR and $C D N K 1 B$ were the only recurring mutations in GI NET. While the frequency of CDKN1B mutations seen in our patient cohort at $5 \%$ was comparable to $8 \%$ reported in the literature (Francis et al. 2013), recurring mutations in $B C O R$ have not been previously reported in SI NET. BCOR (BCL-6-interacting corepressor) is a known interaction partner of histone deacetylases (HDACs) that plays a critical role in maintaining gene expression through DNA methylation pathways (Huynh et al. 2000).

Recurring loss-of-function mutations in the MEN1 gene and frequent $\mathrm{LOH}$ in chromosome 3 have been previously reported in lung NET (Leotlela et al. 2003, Fernandez-Cuesta et al. 2014). While we did not identify any association between PFS and the extent of CIN in lung NET, there was a trend toward prolonged PFS among patients with $\mathrm{LOH}$ in chromosome 3 . The small numbers of lung NET in this study, however, precludes definitive conclusions pending validation with a larger cohort of patients.

Genomic analysis of pNET revealed a distinct association between recurring mutations in the MEN1/DAXX/ATRX genes and CIN patterns in mutually exclusive sets of chromosomes. Mutations in the MEN1/DAXX/ATRX genes were found to be more common in pNET with extensive CIN along with a very distinct pattern of $\mathrm{LOH}$ and gain in mutually exclusive set of chromosomes. This association between mutations in the MEN1/DAXX/ATRX genes and a distinct pattern of chromosomal aberrations in pNET has not been previously reported. However, these results are consistent with published reports showing association of mutations in MEN1, DAXX and ATRX with higher CIN and alternate lengthening of telomeres in different cancer types (Lin \& Elledge 2003, Mirabello et al. 2010, Heaphy et al. 2011, Lovejoy et al. 2012). Further mechanistic studies are warranted to understand how mutations in these genes may be linked to distinct chromosomal aberration patterns in pNET. It is likely that CIN in pNET is the result of instability related to MEN1/DAXX/ATRX mutations. MEN1 has been linked to telomere length and
$D A X X / A T R X$ mutations lead to alternative lengthening of telomeres. The differences in outcome may also reflect differences in MEN1-mutated (associated with high CIN) vs MEN1-WT oncogenesis of pNET (Mirabello et al. 2010, Heaphy et al. 2011).

The three pNET clusters identified in our study corresponds to group 1, 2 and 4 in the study conducted by Scarpa et al. (2017). While, the group 3 (smallest group), consists of a small number of patients with polyploid tumors that were not identified in our current analyses or prior analyses using different techniques (Nagano et al. 2007, Scarpa et al. 2017). The identification of this additional small group in Scarpa et al.'s study could be due to the larger number of pNET patients or due to differences in the study population (Scarpa et al. 2017). The Karpathakis paper addresses small bowel NET and identified three groups, which were similar to our study, wherein, a group with chromosome $18 \mathrm{LOH}$ was identified (Karpathakis et al. 2016). Both these studies identified the alterations in chromosome 4, 5 and 20. The studies seem to have grouped slightly differently based on the difference in patterns on whether chromosome 7 or 14 was involved along with alterations on chromosome 4, 5 and 20 . These may be due to differences in sample size, population and how finely the groups were clustered.

Conflicting results have been previously reported with regard to prognostic role of mutations in the MEN1, DAXX and ATRX genes in pNET. Some studies have suggested a longer OS in pNET with mutations in DAXX and ATRX genes, while others have reported a significantly shorter OS among pNET with the loss of nuclear expression of $D A X X$ and ATRX proteins using immunohistochemistry (IHC) (Jiao et al. 2011, Marinoni et al. 2014). While our data suggest a weak trend toward longer OS in patients whose tumors have mutations in MEN1, DAXX or ATRX, the observed difference was not statistically significant. On the other hand, patients whose tumors were CIN high appeared to have longer OS in pNET, although this difference also did not reach statistical significance. Among patients with favorable prognosis based on lowcirculating CgA and NSE levels, we found that there exists a subset of pNET with high CIN, which demonstrates a very long OS, enabling a better estimation of disease prognosis for clinical decision-making by utilizing genomic biomarkers along with other commonly used circulating biomarkers in pNET. Our frequent losses of chromosome 18 and of chromosome 4, 5 and 20 are supported by the observations of Banck et al. (2013).

One puzzling aspect of our data is that the association of CIN status with survival was different between tumor

This work is licensed under a Creative Commons Attribution-NonCommercial-NoDerivatives 4.0

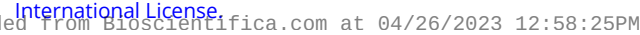


subgroups defined by anatomical locations. In GI NET, low CIN was associated with longer survival, whereas in pNET, low CIN was associated with shorter survival. In lung NET, there was no clear correlation between CIN status and prognosis. This difference of impact of CIN status on survival highlights the different genetic make-up of pNET and GI NET. These observations suggest that while CIN is a common feature among all NET, it may not by itself drive tumor biology and may instead be a reflection of diverse underlying defects in chromosomal maintenance that underlie the development of NET. The limitation of our study was the inability to provide comparison with the finer GI NET tumor subsites due to small sample size.

\section{Conclusions}

Our analyses highlight that large-scale chromosomal gains and deletions are a common feature across all NET subtypes and specific patterns of chromosomal gain and loss appeared to have independent prognostic value in some tumor subtypes. However, whether CIN in general has clinical significance in NET requires validation in larger patient cohort and warrants further mechanistic studies to understand the causes for widespread CIN, which was commonly observed in NET.

\section{Supplementary data}

This is linked to the online version of the paper at https://doi.org/10.1530/ ERC-18-0332.

\section{Declaration of interest}

$\mathrm{J}$ Yao is a consultant and received personal fees from Novartis and Ipsen outside the submitted work. S Singh has received research grants from Novartis and Ipsen, and received personal fees and nonfinancial support from Novartis, Ipsen and Pfizer outside the submitted work. E Van Cutsem has received research grants from Amgen, Bayer, Boehringer, Celgene, Ipsen, Lilly, Merck, Merck KgA, Novartis, Roche, Sanofi and Servier outside the submitted work. R Pommier is a consultant to Novartis and Ipsen, speaker for Novartis, and has received travel and accommodations from both. M Pavel has received personal fees from Novartis, Ipsen, Lexicon and Pfizer; research grants from Novartis and Ipsen outside the submitted work. $\mathrm{M} \mathrm{H}$ Kulke has received personal fees from Lexicon, Novartis and Ipsen. J Capdevila has received grants and personal fees from Novartis, Ipsen and Pfizer outside the submitted work. N Fazio has received grants and personal fees from Novartis and personal fees from Ipsen and Pfizer outside the submitted work. P Engstrom has no financial disclosures. D Chen (during the time of this research) and M Voi are employees of Novartis and holders of Novartis stocks. A Garg (during the time of this research), M Morrissey, M Riester, P Patel and W He are employees of Novartis.

\section{Funding}

The study was sponsored by Novartis Pharmaceuticals Corporation.

\section{Author contribution statement}

J Y, M H K, D C, A G, W H, M M were involved in study concept and design. $J Y, P E, E \vee C, S S, R P, N F, J C, P P$ were involved in data acquisition. J $Y$, $M H K, D C, A G, W H, M M, M R, P P, M V, J C, P E, E V C, S S, R P, M P, N F$ were involved in analysis and interpretation of data. J $Y, M H K, D C, A G$, W H were involved in drafting the manuscript.

\section{Acknowledgements}

The authors would like to thank the patients, their caregivers and investigators for their participation in the study. In addition, they also thank the worldwide network of research nurses, trial coordinators and operations staff for their contributions, and Charu Pundir (Novartis Healthcare Pvt Ltd) for providing medical editorial assistance with this article.

\section{References}

Banck MS, Kanwar R, Kulkarni AA, Boora GK, Metge F, Kipp BR, Zhang L, Thorland EC, Minn KT, Tentu R, et al. 2013 The genomic landscape of small intestine neuroendocrine tumors. Journal of Clinical Investigation 123 2502-2508. (https://doi.org/10.1172/ JCI67963)

Cibulskis K, Lawrence MS, Carter SL, Sivachenko A, Jaffe D, Sougnez C, Gabriel S, Meyerson M, Lander ES \& Getz G 2013 Sensitive detection of somatic point mutations in impure and heterogeneous cancer samples. Nature Biotechnology 31 213-219. (https://doi.org/10.1038/ nbt.2514)

Cingolani P, Platts A, Wang le L, Coon M, Nguyen T, Wang L, Land SJ, Lu X \& Ruden DM 2012 A program for annotating and predicting the effects of single nucleotide polymorphisms, SnpEff: SNPs in the genome of Drosophila melanogaster strain w1118; iso-2; iso-3. Fly 6 80-92. (https://doi.org/10.4161/fly.19695)

Falconi M \& Partelli S 2017 Neuroendocrine tumours in 2016: defining rules for increasingly personalized treatments. Nature Reviews. Clinical Oncology 14 80-82. (https://doi.org/10.1038/ nrclinonc.2016.197)

Fernandez-Cuesta L, Peifer M, Lu X, Sun R, Ozretic L, Seidal D, Zander T, Leenders F, George J, Muller C, et al. 2014 Frequent mutations in chromatin-remodelling genes in pulmonary carcinoids. Nature Communications 5 3518. (https://doi.org/10.1038/ncomms4518)

Frampton GM, Fichtenholtz A, Otto GA, Wang K, Downing SR, He J, Schnall-Levin M, White J, Sanford EM, An P, et al. 2013 Development and validation of a clinical cancer genomic profiling test based on massively parallel DNA sequencing. Nature Biotechnology 31 1023-1031. (https://doi.org/10.1038/nbt.2696)

Francis JM, Kiezun A, Ramos AH, Serra S, Pedamallu CS, Qian ZR, Banck MS, Kanwar R, Kulkarni AA, Karpathakis A, et al. 2013 Somatic mutation of CDKN1B in small intestine neuroendocrine tumors. Nature Genetics 45 1483-1486. (https://doi.org/10.1038/ ng.2821)

Hashemi J, Fotouhi O, Sulaiman L, Kjellman M, Hoog A, Zedenius J \& Larsson C 2013 Copy number alterations in small intestinal neuroendocrine tumors determined by array comparative genomic hybridization. BMC Cancer 13 505. (https://doi.org/10.1186/14712407-13-505)

Heaphy CM, de Wilde RF, Jiao Y, Klein AP, Edil BH, Shi C, Bettegowda C, Rodriguez FJ, Eberhart CG, Hebbar S, et al. 2011 Altered telomeres in tumors with ATRX and DAXX mutations. Science 333 425. (https:// doi.org/10.1126/science.1207313)

Huynh KD, Fischle W, Verdin E \& Bardwell VJ 2000 BCoR, a novel corepressor involved in BCL-6 repression. Genes and Development 14 1810-1823. (https://doi.org/10.1101/gad.14.14.1810) 
Jiao Y, Shi C, Edil BH, de Wilde RF, Klimstra DS, Maitra A, Schulick RD, Tang LH, Wolfgang CL, Choti MA, et al. 2011 DAXX/ATRX, MEN1, and mTOR pathway genes are frequently altered in pancreatic neuroendocrine tumors. Science 331 1199-1203. (https://doi. org/10.1126/science.1200609)

Karpathakis A, Dibra H, Pipinikas C, Feber A, Morris T, Francis J, Oukrif D, Mandair D, Pericleous M, Mohmaduvesh M, et al. 2016 Prognostic impact of novel molecular subtypes of small intestinal neuroendocrine tumor. Clinical Cancer Research 22 250-258. (https:// doi.org/10.1158/1078-0432.CCR-15-0373)

Leotlela PD, Jauch A, Holtgreve-Grez H \& Thakker RV 2003 Genetics of neuroendocrine and carcinoid tumours. Endocrine-Related Cancer 10 437-450. (https://doi.org/10.1677/erc.0.0100437)

Li H 2013 Aligning sequence reads, clone sequences and assembly contigs with BWA-MEM. (available at arXiv:1303.3997v1 [q-bio.GN])

Lin SY \& Elledge SJ 2003 Multiple tumor suppressor pathways negatively regulate telomerase. Cell 113 881-889. (https://doi.org/10.1016/ S0092-8674(03)00430-6)

Lovejoy CA, Li W, Reisenweber S, Thongthip S, Bruno J, de Lange T, De S, Petrini JH, Sung PA, Jasin M, et al. 2012 Loss of ATRX, genome instability, and an altered DNA damage response are hallmarks of the alternative lengthening of telomeres pathway. PLOS Genetics $\mathbf{8}$ e1002772. (https://doi.org/10.1371/journal.pgen.1002772)

Marinoni I, Kurrer AS, Vassella E, Dettmer M, Rudolph T, Banz V, Hunger F, Pasquinelli S, Speel EJ \& Perren A 2014 Loss of DAXX and ATRX are associated with chromosome instability and reduced survival of patients with pancreatic neuroendocrine tumors. Gastroenterology 146 453.e5-460.e5. (https://doi.org/10.1053/j. gastro.2013.10.020)

McKenna A, Hanna M, Banks E, Sivachenko A, Cibulskis K, Kernytsky A, Garimella K, Altshuler D, Gabriel S, Daly M, et al. 2010 The Genome Analysis Toolkit: a MapReduce framework for analyzing nextgeneration DNA sequencing data. Genome Research 20 1297-1303. (https://doi.org/10.1101/gr.107524.110)

Mirabello L, Yu K, Kraft P, De Vivo I, Hunter DJ, Prescott J, Wong JY, Chatterjee N, Hayes RB \& Savage SA 2010 The association of telomere length and genetic variation in telomere biology genes. Human Mutation 31 1050-1058. (https://doi.org/10.1002/ humu.21314)

Nagano Y, Kim DH, Zhang L, White JA, Yao JC, Hamilton SR \& Rashid A 2007 Allelic alterations in pancreatic endocrine tumors identified by genome-wide single nucleotide polymorphism analysis. EndocrineRelated Cancer 14 483-492. (https://doi.org/10.1677/ERC-06-0090)

Pavel ME, Hainsworth JD, Baudin E, Peeters M, Horsch D, Winkler RE, Klimovsky J, Lebwohl D, Jehl V, Wolin EM, et al. 2011 Everolimus plus octreotide long-acting repeatable for the treatment of advanced neuroendocrine tumours associated with carcinoid syndrome (RADIANT-2): a randomised, placebo-controlled, phase 3 study. Lancet 378 2005-2012. (https://doi.org/10.1016/S01406736(11)61742-X)
Riester M, Singh AP, Brannon AR, Yu K, Campbell CD, Chiang DY \& Morrissey MP 2016 PureCN: copy number calling and SNV classification using targeted short read sequencing. Source Code for Biology and Medicine 11 13. (https://doi.org/10.1186/s13029-0160060-z)

Scarpa A, Chang DK, Nones K, Corbo V, Patch AM, Bailey P, Lawlor RT, Johns AL, Miller DK, Mafficini A, et al. 2017 Whole-genome landscape of pancreatic neuroendocrine tumours. Nature 543 65-71. (https://doi.org/10.1038/nature21063)

Sun JX, Frampton G, Wang K, Ross JS, Miller VA, Stephens PJ, Lipson D \& Yelensky R 2014 A computational method for somatic vs. germline variant status determination from targeted Next generation sequencing of clinical cancer specimens without a matched normal control. American Association for Cancer Research Annual Meeting; April 5-9; San Diego, CA. Cancer Research 74 (19 Supplement) abstract 1893. (https://doi.org/10.1158/1538-7445. AM2014-1893)

van Adrichem RC, Kamp K, Vandamme T, Peeters M, Feelders RA \& de Herder WW 2016 Serum neuron-specific enolase level is an independent predictor of overall survival in patients with gastroenteropancreatic neuroendocrine tumors. Annals of Oncology 27 746-747. (https://doi.org/10.1093/annonc/mdv626)

Yao JC, Hassan M, Phan A, Dagohoy C, Leary C, Mares JE, Abdalla EK, Fleming JB, Vauthey JN, Rashid A, et al. 2008 One hundred years after 'carcinoid': epidemiology of and prognostic factors for neuroendocrine tumors in 35,825 cases in the United States. Journal of Clinical Oncology 26 3063-3072. (https://doi.org/10.1200/ JCO.2007.15.4377)

Yao JC, Shah MH, Ito T, Bohas CL, Wolin EM, Van Cutsem E, Hobday TJ, Okusaka T, Capdevila J, de Vries EG, et al. 2011 Everolimus for advanced pancreatic neuroendocrine tumors. New England Journal of Medicine 364 514-523. (https://doi.org/10.1056/ NEJMoa1009290)

Yao JC, Fazio N, Singh S, Buzzoni R, Carnaghi C, Wolin E, Tomasek J, Raderer M, Lahner H, Voi M, et al. 2016a Everolimus for the treatment of advanced, non-functional neuroendocrine tumours of the lung or gastrointestinal tract (RADIANT-4): a randomised, placebo-controlled, phase 3 study. Lancet 387 968-977. (https://doi. org/10.1016/S0140-6736(15)00817-X)

Yao JC, Pavel M, Lombard-Bohas C, Cutsem EV, Voi M, Brandt U, He W, Chen D, Capdevila J, de Vries EGE, et al. 2016b Everolimus for the Treatment of Advanced Pancreatic Neuroendocrine Tumors: Overall Survival and Circulating Biomarkers From the Randomized, Phase III RADIANT-3 Study. Journal of Clinical Oncology 34 3906-3913. (https://doi.org/10.1200/JCO.2016.68.0702)

Ye K, Schulz MH, Long Q, Apweiler R \& Ning Z 2009 Pindel: a pattern growth approach to detect break points of large deletions and medium sized insertions from paired-end short reads. Bioinformatics 25 2865-2871. (https://doi.org/10.1093/ bioinformatics/btp394)

Received in final form 7 December 2018

Accepted 21 January 2019

Accepted Preprint published online 21 January 2019 https://erc.bioscientifica.com https://doi.org/10.1530/ERC-18-0332 (c) 2019 The authors Published by Bioscientifica Ltd. Printed in Great Britain
This work is licensed under a Creative Commons Attribution-NonCommercial-NoDerivatives 4.0

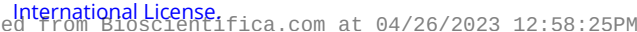

